\section{MODULATION OF TUMOR IMMUNOGENICITY BY DNA METHYLATION OF IMMUNE SYNAPSE GENES IN CANCERS}

Imene Hamaidi*, Anders Berglund, Matthew Mills, Ryan Putney, James Mule, Sungjune Kim. H. Lee Moffitt Cancer Center and Research Institute, Tampa, FL, USA

Background Cancer immunotherapy represents a major paradigm shift in cancer care. Despite such breakthrough, majority of cancer patients remains refractory to existing immunotherapeutic modalities highlighting the inherent capacity of tumors to escape immunosurveillance mechanisms. Frequently, cancer cells utilize the epigenetic machinery to silence tumor suppressors or activate oncogenes for survival and proliferation. Likewise, tumor cells might employ the epigenetic reprogramming of immune-related pathways to evade the immune system. Methylation is one of the major epigenetic mechanisms modulating gene transcription. Thus, we investigated the methylation profile of both co-stimulatory and immune checkpoint genes in cancer.

Methods Data from The Cancer Genome Atlas (TCGA) were used for methylation profiling and RNA-sequencing analysis. Twenty-six epithelial cancer cell lines with more than 3 mock and three 5-azacitidine-treated samples were selected for analysis from the GSE57342 dataset. t-distributed stochastic neighbor embedding (t-SNE) was calculated using 247 probes for the selected 20 genes across all TCGA samples. t-SNE analysis was performed on 8,186 solid tumors and 745 normal adjacent tissues for methylation levels for all probes. For principal component analysis, first and second principal components were used to represent the overall methylation status for 8,931 tumor and normal samples in the TCGA database. Survival analyses were retrieved from a prior publication. ${ }^{1}$

Results We found that methylation profile of immune synapse genes is distinct in tumor versus normal adjacent tissue. Interestingly, our results demonstrate hypermethylation of co-stimulatory genes such as CD40 and hypo-methylation of immune checkpoint genes such as HHLA2 and PDL1 across multiple tumor types in comparison with the normal adjacent tissue. In addition, an inverse correlation between methylation and gene expression was manifest among tumor and normal adjacent tissue, confirming the epigenetic mechanism of gene suppression by gene methylation. Furthermore, we observed a reversal of hypermethylation of the co-stimulatory genes including CD40 by the demethylating agent 5 -azacytidine in the data set of 26 epithelial cancer cell lines. Finally, we found that that hypomethylation of co-stimulatory genes within the immune synapse correlates with functional $\mathrm{T}$ cell recruitment to the tumor microenvironment and is followed by a favorable clinical outcome in melanoma patients.

Conclusions Our finding unveils methylation of immune synapse genes as a crucial driver of the immune evasive phenotype of cancer cells. Notably, identification of actionable targets to restore tumor immunogenicity is an attractive strategy in combination with immune checkpoint blockade.

Acknowledgements This work was supported by NIH grant K08 CA194273, the Immunology Innovation Fund, an NCI Cancer Center Support grant, (P30-CA076292), the Miriam and Sheldon G. Adelson Foundation, and the Moffitt Foundation.

\section{REFERENCE}

1. Liu J, Lichtenberg T, Hoadley KA, Poisson LM, Lazar AJ, Cherniack AD, Kovatich AJ, Benz CC, Levine DA, Lee AV, Omberg L, Wolf DM, Shriver CD, Thorsson V, Cancer Genome Atlas Research N, Hu H. An integrated TCGA pan-cancer clinical data resource to drive high-quality survival outcome analytics. Cell 2018;173 (2):400-16 e11.

http://dx.doi.org/10.1136/jitc-2021-SITC2021.907 